\title{
Situation Épidémiologique des Leishmanioses au Niveau de la Ville d'EI Hajeb (Centre du Maroc) Durant la Période de 2013 à 2017
}

\author{
Karima El-Mouhdi \\ Laboratoire de la géo-biodiversité et patrimoine naturel, centre GEOPAC, \\ institut scientifique-Rabat, Université Mohamed V. Maroc

\section{Abdelkader Chahlaoui} \\ Equipe Gestion et valorisation des ressources naturelles, \\ Laboratoire Santé et environnement, Faculté des Sciences, \\ Université Moulay Ismail, Meknès, Maroc

\section{Abdelhakim El-Ouali Lalami} \\ Institut Supérieur des Professions Infirmières et Techniques \\ de santé de Fès, Maroc

\section{Jawad Bouzid} \\ Institut Supérieur des Professions Infirmières et Techniques \\ de santé de Meknès, Maroc
}

\section{Hajar El Omari}

Equipe Gestion et valorisation des ressources naturelles, Laboratoire Santé et environnement, Faculté des Sciences, Université Moulay Ismail, Meknès, Maroc

\section{Mohammed Fekhaoui}

Institut Scientifique, Rabat, Maroc

\section{Doi: 10.19044/esj.2019.v15n3p155 URL:http://dx.doi.org/10.19044/esj.2019.v15n3p155}

\section{Resume}

Les leishmanioses sont des maladies infectieuses transmises à l'homme par la piqure d'un phlébotome femelle. Elles constituent la deuxième cause de mortalité mondiale d'origine parasitaire après le paludisme et elles sont endémiques dans les pays d'Asie et d'Afrique. Au Maroc, elles posent un vrai problème de santé. L'objectif de cette étude est de déterminer leur situation épidémiologique dans la ville d'EL Hajeb située au centre du Maroc, analyser leur évolution en fonction du sexe et de l'âge et apprécier leur distribution géographique selon le milieu. Pour ce faire, nous avons effectué une étude rétrospective durant la période de 2013 à 2017, colligeant tous les cas des leishmanioses déclarés au niveau de cette ville. Les données ont été collectées au niveau des registres des cas auprès de la délégation du Ministère 
de la santé d'El Hajeb. Au total vingt-un nouveaux cas ont été déclarés infectés. La coexistence des deux formes des leishmanioses: cutanée (80,95\%) et viscérale $(19,05 \%)$ avec une prédominance du milieu rurale $(76,47 \%)$. Seuls les enfants sont concernés par la leishmaniose viscérale et le plus jeune âge était de 13 mois. Le sex-ratio femme/homme était de 1,1. L'âge moyen de contamination est de 3,75 années pour la forme viscérale et de 25,82 années pour la forme cutanée. L'élimination des leishmanioses au Maroc d'ici 2030 nécessite l'adoption d'une approche globale en agissant sur les foyers de contamination par une surveillance et une prise en charge appropriées, une lutte efficace contre les vecteurs et les réservoirs et une innovation dans les stratégies de sensibilisation.

Mots clés: Leishmanioses, Phlébotome, Situation épidémiologique, Etude rétrospective, Centre du Maroc 


\title{
Epidemiological Situation of Leishmaniasis in the City of EI Hajeb (Central Morocco) During the Period 2013-2017
}

\author{
Karima El-Mouhdi \\ Laboratoire de la géo-biodiversité et patrimoine naturel, centre GEOPAC, \\ institut scientifique-Rabat, Université Mohamed V. Maroc \\ Abdelkader Chahlaoui \\ Equipe Gestion et valorisation des ressources naturelles, \\ Laboratoire Santé et environnement, Faculté des Sciences, \\ Université Moulay Ismail, Meknès, Maroc \\ Abdelhakim El-Ouali Lalami \\ Institut Supérieur des Professions Infirmières et Techniques \\ de santé de Fès, Maroc \\ Jawad Bouzid \\ Institut Supérieur des Professions Infirmières et Techniques \\ de santé de Meknès, Maroc
}

Hajar El Omari

Equipe Gestion et valorisation des ressources naturelles, Laboratoire Santé et environnement, Faculté des Sciences, Université Moulay Ismail, Meknès, Maroc

Mohammed Fekhaoui

Institut Scientifique, Rabat, Maroc

\section{Abstract}

Leishmaniasis is an infectious disease transmitted to humans by the bite of female sand fly. These parasitic infections are the second most common cause of parasite mortality worldwide after malaria and are endemic Asian and African countries. In Morocco, they pose a real health problem. The objective of this study is to determine the epidemiological situation of these diseases in the city of El Hajeb in central Morocco, analyze their evolution according to sex and age and assess their geographical distribution according to the environment. To do this, we carried out a retrospective study during the period from 2013 to 2017, collecting all the new cases of cutaneous and visceral leishmaniasis declared in this city. Data were collected at the register level from the delegation of the Ministry of health of El Hajeb. A total of twentyone new cases were declared infected. The coexistence of the two forms of leishmaniasis: $80.95 \%$ of cutaneous forms and $19.05 \%$ of the visceral form 
declared only in children. The rural population is the most concerned $(76.47 \%)$ the sex ratio between men women was around 1.1. the average age of contamination by the visceral form is 3.75 years by the cutaneous form. The elimination of leishmaniasis in Morocco by 2030 requires the adoption of a global approach by acting on the foci of contestation through regular surveillance, integrated management of actions against vector and reservoirs and an innovation in sensibilisation strategy.

Keywords: Leishmaniasis, Sand flay, Epidemiological situation, Retrospective study, Central Morocco

\section{Introduction}

Les leishmanioses sont des maladies parasitaires largement répandues à la surface de la terre, elles possèdent une aire de répartition globalement circumterrestre (Dedet, 2001; Pilly, 2015). Elles représentent un sérieux problème de santé dans les pays tropicaux en raison de leur diversité clinique (cutanée, viscérale et cutanée-muqueuse) et épidémiologique, de la complexité de leurs cycle parasitaires et de la multiplicité de leurs réservoirs (homme, rongeur et chien). Ces maladies sont endémiques dans la majorité des pays ayant un climat tempéré comme le Soudan, le Maroc, l'Algérie, la Tunisie, le Bengladesh, 1'Inde et le Brésil (Traoré et al., 2001; Gutiérrez et al., 2017; Houti et al., 2016 ; Fendri, et al., 2012 ; Aoun,et al., 2009 ; Singh et al., 2012). Elles sont transmises à l'homme par la piqure d'une petite mouche de sable infestée: phlébotome femelle, ce moucheron est bien adapté aux climats tropicaux et subtropicaux et sont répondus dans la région méditerranéenne (Gutiérrez et al., 2017 ; Tiwari, et al. 2018) ; Duvallet, 2015 ; Haouas et al., 2015). Les leishmanioses se manifestent sous différentes formes : viscérale, cutanées ou cutanéo-muqueuses et ont des territoires dont la délimitation dépend de facteurs intrinsèques liés aux espèces de parasite, de phlébotomes vecteurs et de mammifères réservoirs, mais également de facteurs extrinsèques, environnementaux, notamment l'insalubrité des espaces (Houti et al., 2016), . La prévalence mondiale dépasse 10 million de cas, il y a 1,5-2 nouveaux cas annuels, dont une forte proportion d'enfants et la mortalité mondiale annuelle est de 70000 soit la deuxième cause de mortalité d'origine parasitaire après le paludisme, le poids est de 2,4 million d'AVCI (Années de Vie Corrigées de 1'Incapacité) (Pilly, 2015).

$\mathrm{Au}$ Maroc, les leishmanioses sont le premier groupe de maladie à transmission vectorielle et faisant partie des maladies infectieuses à déclaration obligatoire selon l'arrêté ministériel N 683-95 du 31 mars 1995. Malgré cette réglementation, ces infections posent un vrai problème de santé, en raison du nombre croissant des cas dépistés chaque année et de l'extension de la leishmaniose cutanée à d'autres zones auparavant indemne (El Alami, 
2009; Houti et al., 2016 ; Boussaa, 2014) . C'est dans ce contexte ont été réalisées plusieurs études au niveau des villes marocaines : la ville de Tanant (Arroub, 2016), de Beni Mellal (Zouirech et al., 2015), de Boulmane (Talbi et al., 2015), de Moulay Yacoub (Lahouiti, et al., 2013), de Meknès (ELOmari et al., 2016), d'Azilal (Zouirech et al., 2015); de Marrakech (Boussaa et al., 2014) et la région d'El Gharb Chrarda Beni Hassan (El Aasri et al., 2016). A notre connaissance, aucune étude concernant la situation épidémiologique des cas des leishmanioses n'a été faite au niveau de la ville d'EL hajeb située limitrophe des zones endémiques : Fès et Meknès. Ainsi, une étude épidémiologique des cas des leishmanioses serait nécessaire et indispensable pour déterminer et explorer la situation de ces parasitoses au niveau de la province d'El Hajeb. Les résultats de cette étude seront d'un grand intérêt pour les services d'hygiène impliqués dans la lutte anti vectorielle, au niveau local qu'au niveau central, pour mieux mener les actions de lutte et de prévention.

\section{Matériels et méthodes}

- Zone d'étude

La zone d'étude est la ville d'El Hajeb se située a centre du Maroc et elle appartient à la région Fès-Meknès. Elle est limitée à l'Est par la préfecture de Fès, à l'Ouest par la province de Khémissate, au Nord par la préfecture de Meknès et au Sud par la province d'Ifrane. Sa superficie est de $2208 \mathrm{~km}$ et ses coordonnées géographiques sont : Longitude $5^{\circ} 22^{\prime}$, Latitude $33^{\circ} 41^{\prime}$ et Altitude $1000 \mathrm{~m}$. En effet, la ville d'El Hajeb s'allonge sur les premiers contreforts de la chaîne montagneuse du Moyen Atlas, on trouve 16 communes dont 12 rurales et leurs habitants constituées principalement par les tribus : Bni Mtir, à raison de 75\% et Gerouane sud, à raison de 25\% (Ouard et al., 2011). Ainsi, le nombre total de la population d'El hajeb est estimé selon le dernier recensement général de la population et de l'habitat de 2014 à 247016 habitants (Haut Commissariat au Plan, 2014).

\section{- Matériels et méthodes}

L'étude a intéressé tous les nouveaux cas des leishmanioses cutanées (LC) et des leishmanioses viscérales (LV) enregistrés depuis le $1^{\text {er }}$ Janvier 2013 jusqu'au 31 Décembre 2017. En effet, les données de cette étude ont été relevées à partir de données publiées officiellement dans les rapports de la santé en chiffre des années 2013 à 2017 (Ministère de la santé Marocaine, 2014, 2018). Cependant, ces documents rapportent seulement le nombre total des cas des leishmanioses déclarés à la ville d'El Hajeb sans distinction entre les type des leishmanioses ni les caractéristiques sociodémographiques des personnes contaminées tels que : l'âge, le sexe, le milieu de vie (rural ou urbain), la classification des cas (autochtone, importé, paradoxal) et le type de dépistage (actif ou passif). Pour ceci, nous avons complété les données de 
notre étude en les recueillant au niveau des registres des cas auprès de la délégation du Ministère de la santé d'El Hajeb. Nous avons, ensuite, calculé le taux d'incidence des leishmanioses qui correspond au nombre de nouveaux cas dépistés pour cent mille habitant dans une population exposée donnée en une année.

L'analyse statistique a été entreprise pour l'ensemble des données qui ont été saisis et regroupés sur une grille d'Excel (Microsoft) en fonction de l'âge, du sexe, du milieu et du type de la leishmaniose (LC ou LV). Le calcul des totaux, moyennes, médianes et intervalles a été effectué pour les variables quantitatives tandis que les pourcentages ont été déterminés pour celles qualitatives. La comparaison deux pourcentages a été faite manuellement à l'aide logiciel Epi Info7.

\section{Résultats}

- L'incidence des cas

Au total 21 nouveaux cas des LC et LV ont été enregistrés entre le $1^{\text {er }}$ Janvier 2013 et 31 Décembre 2017 à la ville d'El Hajeb. L'analyse de l'évolution annuelle des cas des leishmanioses en fonction des communes de la ville d'El Hajeb durant les cinq dernières années (figure 1) montre une très forte prédominance des communes rurales qui représentait environ les trois quart des communes touchées par les leishmanioses. En 2013 et 2014, la commune la plus touchée par ces parasitoses est celle de Laqsir avec un taux d'incidence de $1,62 \%$ et 1,41\%. En 2015, c'est la commune d'Ain Taoujdate qui est la plus concernée avec un taux d'incidence de 0,21\%. En 2016, ce sont les deux communes rurales de Bitit et Ait Ouikhlfen qui ont été exposées avec un taux d'incidence pour chacune de 0,4\%. En 2017, ce sont les communes de Bitit, Tamchachate et El Hajeb qui ont été touchées avec un taux d'incidence pour chacune de $0,61 \%$.

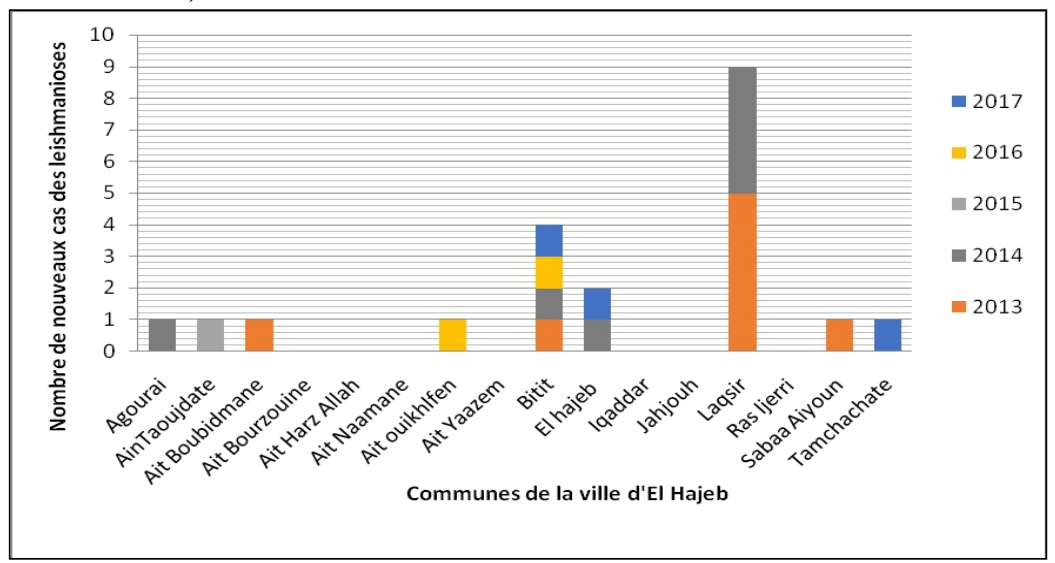

Figure 1: Évolution annuelle des nouveaux cas des leishmanioses en fonction des communes de la ville d'ELHajeb (2013-2017) 


\section{- La répartition des nouveaux cas en fonction de l'âge, du sexe et du} type de la leishmaniose

L'analyse de la répartition des nouveaux cas en fonction de l'âge et du sexe et du type des leishmanioses (Tableau 1) montre que l'âge moyen était 3,75 années pour la LV et 25,82 années pour la LC. Le sex-ratio homme/femme était 1,1 avec une prédominance du sexe féminin à l'âge adulte.

Chez la population d'EL Hajeb, l'analyse de la répartition des nouveaux cas en fonction du type des leishmanioses: cutanées (LC) viscérale (LV) montre la prédominance de la forme cutanée, qui représentait environ $81 \%$ des cas identifiés. Chez la population d'adulte, l'infestation par la LV est absente alors que celle par la LC représentait $14,28 \%$ pour l'homme et $23,8 \%$ pour la femme.

Chez la population infantile est la catégorie la plus touchée par ces parasitoses avec un taux de 61,9\%. Ainsi, l'infestation par la forme cutanée représentait $23,81 \%$ chez la tranche d'âge de 5 à 14 ans et $19,05 \%$ chez la tranche d'âge de 0 à 4 ans. Cependant, la forme viscérale a été déclarée seulement chez cette population et représentait trois quarts des cas chez la petite enfance âgée de 0 à 4 ans et le reste enregistré chez les enfants âgés de 5 à 14 ans.

Tableau 1: Pourcentage des cas des leishmanioses cutanées et viscérales en fonction de l'âge et du sexe pendant la période de 2013 à 2017

\begin{tabular}{|c|c|c|c|c|c|c|c|c|c|c|}
\hline & \multicolumn{2}{|c|}{$\begin{array}{l}\text { Total } \\
n=21(100 \%)\end{array}$} & \multicolumn{2}{|c|}{$\begin{array}{l}\text { Enfants [0-4ans] } \\
n=7(33,33 \%)\end{array}$} & \multicolumn{2}{|c|}{$\begin{array}{l}\text { Enfants [5-14ans] } \\
n=6(28,57 \%)\end{array}$} & \multicolumn{2}{|c|}{$\begin{array}{l}\text { Femmes } \geq 15 \text { ans } \\
n=5(23,8 \%)\end{array}$} & \multicolumn{2}{|c|}{$\begin{array}{l}\text { Hommes } \geq 15 \text { ans } \\
n=3(14,28 \%)\end{array}$} \\
\hline & $\begin{array}{l}\text { Nombre } \\
\text { des } \\
\text { N.cas* }\end{array}$ & $\%$ & $\begin{array}{l}\text { Nombre } \\
\text { des N.cas }\end{array}$ & $\%$ & $\begin{array}{l}\text { Nombre } \\
\text { des N.cas }\end{array}$ & $\%$ & $\begin{array}{l}\text { Nombre } \\
\text { des N.cas }\end{array}$ & $\%$ & $\begin{array}{l}\text { Nombre } \\
\text { des N.cas }\end{array}$ & $\%$ \\
\hline $\begin{array}{l}\text { Leishmaniose } \\
\text { Cutanée }\end{array}$ & 17 & 80,95 & 4 & 19,05 & 5 & 23,81 & 5 & 23,81 & 3 & 14,29 \\
\hline $\begin{array}{c}\text { Leishmaniose } \\
\text { Viscérale }\end{array}$ & 4 & 19,05 & 3 & 14,29 & 1 & 4,76 & 0 & 0 & 0 & 0 \\
\hline
\end{tabular}

*N.cas : Nouveaux cas

\section{- La distribution géographique des nouveaux cas}

La distribution géographique des nouveaux cas des leishmanioses en fonction du milieu (figure 2). La répartition de ces pathologies est en faveur du milieu rural qui représentait plus de trois quarts des cas identifiés, soit $76,47 \%$ pour la LC et $75 \%$ pour la LV. Les nouveaux cas autochtones représentaient un tiers des cas dépistés ( 7 cas). Le dépistage actif a été pratiqué seulement pour identifier les nouveaux cas de LC $(11,76 \%)$ et importés $(9,52 \%)$. Ainsi, aucun nouveau cas de LV ou autochtone n'a été identifié par ce type de dépistage. 


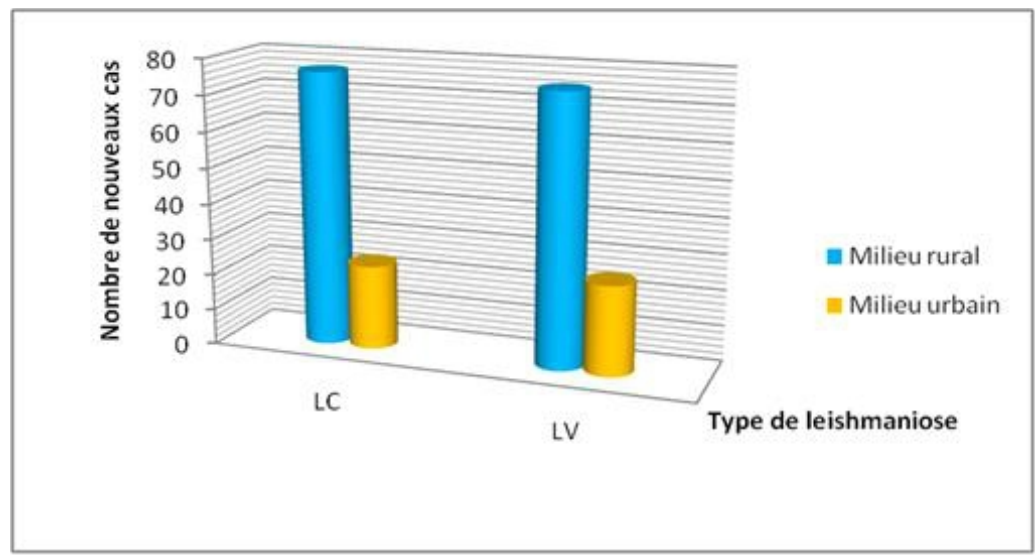

Figure 2: Répartition des nouveaux cas des leishmanioses selon le milieu urbain et rural

\section{- La répartition mensuelle des nouveaux cas}

La répartition des nouveaux cas des LC et de LV sur les 12 mois de l'année est présentée sur la (figure3). La déclaration des nouveaux cas de la LC a été faite principalement toute l'année sauf les mois de Juin, Juillet et Août. Tandis que l'identification des nouveaux cas de la LV était présente durant les mois d'Avril, Octobre et Novembre.

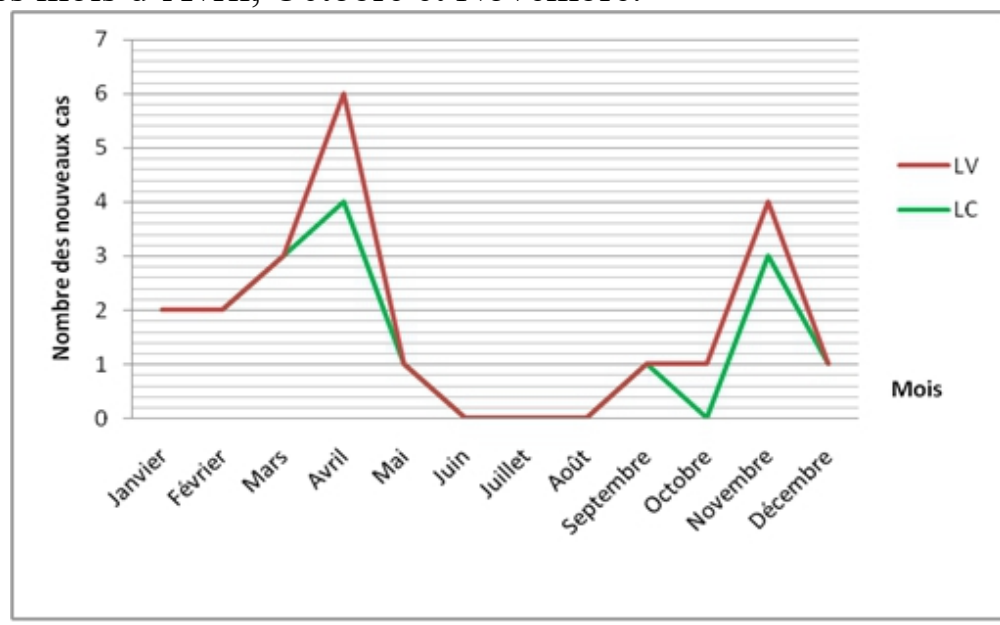

Figure 3: Distribution des nouveaux cas des leishmanioses sur les 12 mois de l'année

\section{Discussion}

Actuellement, la leishmaniose est considérée par l'OMS comme un groupe de maladies tropicales les plus négligées dans le monde. Elle touche des millions des personnes dans le pays sous développés en laissant des cicatrices indélébiles et défigurantes et tue des milliers d'autres dont la majorité entre eux est les petits enfants. Se sont des pathologies zoonotiques communes à l'homme et aux animaux. Elles sont considérées comme des maladies tropicales négligées qui attaquent les viscères, les muqueuses et la 
peau de l'homme, elles touchent les populations pauvres et pèsent lourdement sur les systèmes de santé dans les pays sous développés et en voie de développement (Chan, 2009).

$\mathrm{Au}$ Maroc, la leishmaniose est la première maladie à transmission vectorielle, elle est déclarée par le Ministère de la santé comme une zoonose majeure dans sa forme viscérale et un vrai problème de santé publique dans sa forme cutanée. Ces pathologies s'étendent de forme cutanée bénigne à la forme viscérale de pronostic grave : en moyen 150 nouveaux cas de la leishmaniose viscérale à Leishmania infantum sont identifiés annuellement dans les centres hospitaliers soit un taux de 3\% et les leishmanioses cutanées qui sont marquées par une évolution endémo-épidémique représentent en moyen 4773 nouveaux cas déclarés chaque année avec un pourcentage de 97\% (Ministère de la santé Marocaine, 2014, 2018).

Au niveau d'El Hajeb et durant les cinq années 2013-2017, la maladie a été observée chez la population au cours des mois Mars-Avril et Novembre dont les taux les plus forts ont été enregistrés pour la forme cutanée et durant les mois d'Octobre-Novembre et Avril pour la forme viscérale ce qui couvre les saisons de printemps et de l'automne. Ceci pourrait être expliqué par le climat semi continental de type méditerranéen d'El Hajeb qui se caractérise par des saisons d'étés chauds et secs et des hivers froids qui assure un environnement favorable pour le développement de la végétation et constitue des biotopes propices à la multiplication des réservoirs et des vecteurs. En effet, de nombreux auteurs ont, sur la base des indicateurs climatologiques et environnementaux, affirmé les liens qui existent entre les facteurs climatiques et l'incidence des leishmanioses (Githeko et al., 2001; Duvallet, 2006; Houti et al., 2016. Ainsi, les résultats de ce travail se corroborent avec ceux de Rioux et al., (1986) qui ont confirmés que l'infestation vectorielle a augmenté au cours de la saison chaude et nulle en Juin, la période à risque maximale se situe en début d'automne.

En effet, les résultats de notre étude confirment l'existence des deux principales formes des leishmanioses au niveau de la ville d'El Hajeb. Cependant, le pourcentage des cas de la LV $(19,05 \%)$ est plus élevé par rapport à celui qui a été enregistré à l'échelle national (3\%). Ainsi, la forme cutanée est la plus dominante chez la population marocaine est aussi dominante chez la population de notre étude. Ceci pourrait être expliqué, d'une part, par la situation épidémiologique actuelle des leishmanioses au niveau du Royaume devenu préoccupante en raison de la morbidité croissante et de l'identification permanente des nouveaux foyers de transmissions actives ou la réactivité des autres foyers dans des localités périurbaines et rurales où les facteurs de risques persistent (El Alami, 2009 ; (Ministère de la santé Marocaine, 2010 et 2018; Boussaa et al., 2014; OMS, 2016). 
Et d'autre part, par le caractère rural des habitants de la ville d'El Hajeb et leurs habitudes socioculturelles dans la gestion de leur environnement de vie. En fait, l'élimination non hygiéniques des excrétas, la défécation à l'air libre des hommes et des animaux, la présence des points noirs où ils s'accumulent les ordures et les déchets sont des facteurs favorisant la contamination et l'infestation par des maladies parasitaires telles que les leishmanioses. Aussi, la majorité des ruralistes possèdent des chiens puisqu'ils sont très bénéfiques pour la garde des maisons et des champs, mais ils constituent l'un des principaux réservoirs de la Leishmania infantum (Chan, 2009; OMS, 2011). Ainsi, l'Organisation Mondiale de la Santé annonce dans son rapport de la lutte contre les leishmanioses de 2011 qu'il ne faut pas sousestimer le rôle éventuel de ces animaux dans le cycle de transmission parce que $50 \%$ des chiens parasités sont des porteurs asymptomatiques (OMS, 2011).

La répartition des nouveaux cas des leishmanioses en fonction de l'âge montre que les enfants sont la catégorie qui paye lourdement à cause de ces parasitoses, ils sont les seuls concernés par la LV puisque le plus jeune enfant est un nourrisson de 13 mois touché par la forme viscérale et de 2 ans par la forme cutanée ceci montre l'incidence élevé et la gravité de ces zoonoses dans cette ville. Ces résultats se concordent avec réalisés en Algérie (Fendri et al., 2012) et en Tunisie (Aoun et al., 2009) qui ont affirmé que les enfants représentent une population à risque et sont les plus touchés par la forme viscérale. Dans la présente étude, la majorité des patients provenait des zones rurales (76,47\% des cas) où les habitats sont insalubres et constituent un milieu favorable pour la transmission de la maladie, ce qui justifié nos résultats. En effet, dans les zones rurales où vivent la plupart des patients atteints des leishmanioses ( $\mathrm{LC}$ et LV), les habitations et leurs abords constituent des microfoyers permettant les contacts entre l'homme et le phlébotome et assurant ainsi les conditions favorables à la circulation du parasite (Dedet, 2001; Aoun et al., 2009 ; OMS, 2011). Ces résultats se corroborent avec celles de Rioux et al. (1986); de Rhajaoui (2011); de Talbi et al. (2015) et de Lahouiti et al.(2013).

En 2016, le Maroc a été classé par l'OMS parmi les pays à forte charge de morbidité de LC et LV avec un taux d'incidence de 5,62 pour la LC et 0,91 pour la LV avec une population à risque de $14 \%$ pour la $\mathrm{LC}$ et $10 \%$ pour la LV (OMS, 2016). Les résultats de la présente étude affirme l'existence de ces parasitoses au niveau de la zone d'étude d'El Hajeb en raison de la déclaration confirmée des nouveaux cas autochtones des LC et LV dans les communes rurales et urbaines de la ville, ceci pourrait s'expliquer par la propagation des leishmanioses au Maroc en général et par la situation géographique d'El Hajeb en particulier car elle se trouve près des villes Fès et Meknès déclarés récemment comme des foyers actifs des leishmanioses. Ainsi, ces résultats se 
corroborent avec ceux d'EL Omari et al. (2016) et Zouirech et al. (2015) qui ont annoncé la présence des leishmanioses au centre du pays et l'extension de la transmission de ces infections à d'autres aires géographiques.

\section{Conclusion}

$\mathrm{Eu}$ terme de cette étude rétrospective des données de la surveillance épidémiologique concernant l'ensemble des cas autochtones dépistés au niveau d'El Hajeb durant 2013 à 2017, nous concluons la présence des ces maladies sous les deux principales formes : cutanée et viscérale. La déclaration de ces nouveaux cas dans la majorité des communes de la ville est en faveur de l'hypothèse de la propagation d'une éventuelle épidémie de ces parasitoses.

Dans cette optique, d'autres études devront être menées: entomologique pour identifier les vecteurs incriminés dans la transmission (phlébotomes femelles) et mammalogique pour déterminer les réservoirs possibles (chiens et rongeurs). Aussi, d'autres approches devront être adoptées : sociologique pour savoir les représentations sociales des patients atteints des lésions cutanées inesthétiques et viscérales graves causées par une simple piqure d'un petit insecte et anthropologique pour découvrir les relations homme-insecte et les différents modes de prévention et de pratiques traditionnelles utiliser par la population pour le soin et traitement des lésions cutanées et viscérales. Ainsi, le mariage entre ces approches notamment écoépidémiologique et socio-anthropologique permet de prendre la problématique des leishmanioses dans sa globalité et par conséquent de réussir son élimination d'ici 2030 au niveau national et international en déchiffrant la relation homme-insecte-réservoir, en ciblant les actions de lutte contre les vecteurs et le réservoir et en innovant les mesures de prévention qui doivent être mises en place et mise en œuvre d'une façon interdisciplinaire et multidisciplinaire pour éviter tout risque sanitaire grave qui pourrait être engendré par ses parasitoses.

\section{References:}

1. Aoun, K., Jeddi, F., Amri, F., Ghrab, J., \& Bouratbine, A. (2009). Actualités épidémiologiques de la leishmaniose viscérale en Tunisie. Médecine et MaladiesInfectieuses, 39(10), 775-779. https://doi.org/10.1016/j.medmal.2009.08.010

2. ARROUB, H. (2016). Répartition spatio-temporelle de la leishmaniose cutanée dans les zones semi-arides Marocaines. Consulté à l'adresse https://www.researchgate.net/publication/290391885

3. Boussaa, S., \& Boumezzough, A. (2014). Identification et caractérisation des gîtes larvaires de phlébotomes (Diptera: Psychodidae) à Marrakech (Maroc), 9. 
4. Chan, D. M. (2009). Ces maladies ont une importance internationale dans des secteurs qui vont bien au-delà de la santé”. Maladies tropicales négligées, succès ignorés, nouvelles opportunités.

5. Dedet, J. P. (2001). Répartition géographique des leishmanioses. Médecine et Maladies Infectieuses, 31, 178-183. https://doi.org/10.1016/S0399-077X(01)80057-3

6. Duvallet, G. (2006). Parasites, vecteurs de pathogènes et changements climatiques. Hydroécologie Appliquée, 15, 87-96. https://doi.org/10.1051/hydro: 2006008

7. El Aasri, A., Alaoui, Z., El Kharrim, K., Belghyti, D., Aqachmar, Y., \& Hadji, M. (2016). Profil Epidemiologique De La Leishmaniose Cutanee Dans La Region Du Gharb- Maroc De 2006 À 2014. European Scientific Journal, 12(3). https://doi.org/10.19044/esj.2016.v12n3p243

8. El Alami Sanae. (2009). 85 années de leishmaniose au Maroc. Université Mohamed V de Rabat. Faculté de Medecine et de Pharmacie.

9. Fendri, A. H., Beldjoudi, W., Ahraou, S., \& Djaballah, M. (2012). Les leishmanioses diagnostiquées au CHU Benbadis de Constantine (Algérie): bilan de cinq années (2006-2010). Bulletin de la Société de pathologie exotique, 105(1), 46-48. https://doi.org/10.1007/s13149011-0203-z

10. Gérard Duvallet. (2015). Changement climatique et écologie vectorielle. Bulletin de l'Académie Vétérinaire de France, (2), 116. https://doi.org/10.4267/2042/56861

11. Githeko, A. K., Lindsay, S. W., \& Confalonieri, U. E. (2001). Changement climatique et maladies à transmission vectorielle : une analyse régionale, (4), 11.

12. Gutiérrez-Rebolledo, G. A., Drier-Jonas, S., \& Jiménez-Arellanes, M. A. (2017). Natural compounds and extracts from Mexican medicinal plants with anti-leishmaniasis activity: An update. Asian Pacific Journal of Tropical Medicine, 10(12), 1105-1110. https://doi.org/10.1016/j.apjtm.2017.10.016

13. Haouas, N., Amer, O., Ishankyty, A., Alazmi, A., \& Ishankyty, I. (2015). Profile and geographical distribution of reported cutaneous leishmaniasis cases in Northwestern Saudi Arabia, from 2010 to 2013. Asian Pacific Journal of Tropical Medicine, 8(4), 287-291. https://doi.org/10.1016/S1995-7645(14)60332-1

14. Haut Commissariat au Plan. (2014). POPULATION LÉGALE DES RÉGIONS, PROVINCES, PRÉFECTURES, MUNICIPALITÉS, ARRONDISSEMENTS ET COMMUNES DU ROYAUME D’APRÈS LES RÉSULTATS DU RGPH 2014 (12 Régions). Haut 
Commissariat au Plan du Maroc. Consulté à l'adresse [Online] Availablehttp://www.hcp.ma/Etudes_r161.html

15. Houti, L., Belgat, S., Ikhlef-Allal, A., Makhlouf, B., Hamedi, M., Rahou, A., Bounoua, L. (2016). Impact du climat sur le développement de la leishmaniose cutanée dans la zone humide du Chott Ech Chergui, 17.

16. Lahouiti, K., Lalami, A. E. O., Maniar, S., \& Bekhti, K. (2013). Seasonal fluctuations of phlebotomines sand fly populations (Diptera: Psychodidae) in the Moulay Yacoub province, centre Morocco: Effect of ecological factors. Afr. J. Environ. Sci. Technol., 9.

17. Ministère de la santé Marocaine. (2014). Résumé Stratégie sectorielle 2012-2016.pdf. Established by AMFYMAZ Corporation in Rabat 20.08.2014.

18. Ministère de la santé Marocaine. (2016). SANTE EN CHIFFRES 2013-2017. EDITION 2014-2018.

19. Ministère de la santé Marocaine. (2018). Bulletin d'épidémiologie et de santé publique (Juillet 2018). Volume 56. N 76-1.pdf. Consulté à l'adresse ISSN: 08518238

20. Ministère de la santé Marocaine. Direction de l'Epidémiologie et de Lutte contre les Maladies. Lutte contre les Leishmanioses. Guides des activités 2010.

21. EL Omari, H., Chahlaoui, A., Bouzid, J., \& El Ouali Lalami, A. E. O. (2016). [ Incidence of Cutaneous Leishmaniasis In Meknes Prefecture (Centre of Morocco): A retrospective study of 56 cases collected between 2009 and 2013 ], 18(1), 9.

22. OMS. (2011). La Lutte Contre Les Leishmanioses Rapport De La Reunion Du Comtie OMS D'experts De La Lutte Contre Les Leishmanioses. Organisation Mondiale de la santé. (2016, juin 3). Relevé épidémiologique hebdomadaire. OMS. Consulté à l'adresse ISSN 0049-8114

23. Ouard, A., Merizak, M., \& Mahdi, M. (2011). Gouvernance et acteurs de développement local dans la région de Meknès-Tafilalet Le cas de l'INDH dans la province d'El Hajeb Rapport scientifique.

24. Pilly, E., \& Collège des universitaires de maladies infectieuses et tropicales (France). (2015). Maladies infectieuses et tropicales. Paris: Alinéa Plus.

25. Rhajaoui, M. (2011). Les leishmanioses humaines au Maroc: une diversité nosogéographique. Pathologie Biologie, 59(4), 226-229. https://doi.org/10.1016/j.patbio.2009.09.003

26. Rioux, J., Lanotte, G., Ashford, R., Dereure, J., Petter, F., Akalay, O., ... Moreno, G. (1986). Les leishmanioses cutanées du bassin Méditerranéen occidental. De l'identification enzymatique à l'analyse 
éco-épidémiologique. L'exemple de trois " foyers », tunisien, marocain et français. In Leishmania. TAXONOMIE-PHYLOGENESE (Institut Méditernanéen d'Etude Epidémiologiques et Ecologiques). Montpellier: Jean-Antoine Rioux.

27. Singh, N., Kumar, M., \& Singh, R. K. (2012). Leishmaniasis: Current status of available drugs and new potential drug targets. Asian Pacific Journal of Tropical Medicine, 5(6), 485-497. https://doi.org/10.1016/S1995-7645(12)60084-4

28. Talbi, F., Faraj, C., EL-Akhal, F., ElKhayyat, F., Chenfour, D., ElOualiLalami, A., \& JanatiIdrissi, A. (2015). Diversity_and_Dynamics_of_Sand_Flies_Diptera_Psych.pdf.

Hindawi Publishing Corporation International Journal of Zoology. Consulté à l'adresse http://dx.doi.org/10.1155/2015/497169

29. Tiwari, N., Kishore, D., Bajpai, S., \& Singh, R. (2018). Visceral leishmaniasis: An immunological viewpoint on asymptomatic infections and post kala azar dermal leishmaniasis. Asian Pacific Journal of Tropical Medicine, 11(2), 98. https://doi.org/10.4103/19957645.225016

30. Traoré, K. S., Sawadogo, N. O., Traoré, A., Ouedraogo, J. B., Traoré, K. L., \& Guiguemdé, T. R. (2001). Étude préliminaire de la leishmaniose cutanée dans la ville de Ouagadougou de 1996 à 1998., 4.

31. Zouirech, M., Faraj, C., \& Belghyti, D. (2015). Entomological and epidemiological investigations of an emerging focus of cutaneous leishmaniasis in Bzou, Morocco, 11. 